\title{
Sensing anisotropic stresses with ferromagnetic nanowires
}

\author{
L. Forzani" ${ }^{1 \#}$, A. M. Gennaro ${ }^{1,2}$, R.R. Koropecki ${ }^{1}$, C.A. Ramos ${ }^{3^{*}}$ \\ ${ }^{1}$ Instituto de Física del Litoral, UNL-CONICET, Güemes 3450, 3000 Santa Fe, Argentina \\ ${ }^{2}$ Departamento de Física, Facultad de Bioquímica y Ciencias Biológicas, Universidad Nacional del \\ Litoral (UNL); 3000 Santa Fe, Argentina. \\ ${ }^{3}$ Instituto de Nanociencia y Nanotecnología, CNEA, CONICET, Centro Atómico Bariloche, 8400, S. C. \\ Bariloche, Argentina *Corresponding author e-mail address: cramos@cab.cnea.gov.ar \\ \#Present address: Centro de Investigación de Métodos Computacionales, UNL-CONICET, Santa \\ Fe, Argentina.
}

\begin{abstract}
We have measured the temperature variation of the magnetic anisotropy of Ni nanowires (Ni NW) embedded in freestanding porous anodized aluminum oxide membranes, using DC magnetometry and ferromagnetic resonance. Both techniques show a significant reduction of the uniaxial anisotropy with decreasing temperature. This decrease can be explained by magnetoelastic effects, as Ni NW are subjected to stress due to the difference in thermal expansion coefficients between the nanocomposite materials. Matching our experimental findings with previously measured thermal strains along the $\mathrm{Ni}$ NW axis led us to estimate the perpendicular stress. Thus, we postulate the Ni NW as nanometric differential stress sensors.
\end{abstract}


During the last decades advances in fabrication methods have enabled the development of magnetic structures in which the dimensions have been continuously decreased. Ferromagnetic nanowires are materials that have been extensively studied due to their potential applications in spintronics, magnetic memories, and biosensors among other areas [1-8]. The production of nanocomposites based on porous anodized aluminum oxide (AAO) as a template or matrix structure has the advantage of going beyond the typical size limits of e-beam lithographic designs, and it is scalable to large areas [9-11]. In order to produce the nanowires, the AAO pores are filled with ferromagnetic materials via electrochemical deposition [8, 12]. The magnetic behavior of ferromagnetic nanowires (NW) inside these nanoporous membranes is partly determined by their aspect ratio, dipolar interactions, and crystalline structure [13-16]. Usually, they exhibit uniaxial shape anisotropy with the easy axis parallel to the NW $[13-15,17]$. The magnetic behavior of the NW is also strongly dependent on the mechanical stresses to which the NW are subjected [17]. Dubois et al. [18] studied the behavior of Ni NW electrodeposited into polycarbonate membranes of low porosity. They found an increase of the magnetic anisotropy with decreasing temperature $(T)$ and related this effect to their XRD measurements showing a significant compression along the NW axis at low $\mathrm{T}$. They concluded that magnetoelastic effects caused the observed behavior. They also calculated the radial-to-axial stress ratio and showed it to be negligible in their case [18]. Navas et al. [13], and Sousa et al. [19] reported a decrease of the magnetic anisotropy with decreasing $T$ for $N i N W$ in AAO supported on the native Al substrate. Both results $[13,18]$ were explained by magnetoelastic effects caused by the stress induced by the different thermal expansion coefficients between the components forming the system, attributing the main role to the aluminum substrate, which was the most massive material in their samples.

In a recent XRD study of Ni NW-AAO membranes in which the aluminum substrate had been removed [20] we found an effective negative thermal expansion of the nanocomposite, leading us to conclude that the freestanding AAO also has a negative thermal expansion coefficient in the direction of the pore axes. In this work, we present the temperature dependence of the magnetic properties of the freestanding nanocomposite $\mathrm{Ni}$ NW-AAO, measured using DC magnetometry and ferromagnetic resonance. In the analysis of our results we account quantitatively for the main magnetoelastic contribution - 
along the wire axis- and this allows us to estimate the radial stress exerted by the AAO matrix on the Ni NW.

AAO was produced by a two-step anodization process of electropolished pure Al foil (purity $99.999 \%$ ), using oxalic acid $0.3 \mathrm{M}$ at $280 \mathrm{~K}$ with an anodization voltage of $40 \mathrm{~V}$ to obtain a matrix with pores of $40 \mathrm{~nm}$ in diameter and a distance between pores of $120 \mathrm{~nm}$ [21] (Supplementary Material). The voltage after the second anodization was decreased exponentially from $40 \mathrm{~V}$ to $8.3 \mathrm{~V}$ in order to reduce the thickness of the insulating barrier layer between $\mathrm{AAO}$ and $\mathrm{Al}$ substrate, to allow electron transport during $\mathrm{Ni}$ electrodeposition. We estimate that the barrier thickness was reduced to the optimum value of $10 \mathrm{~nm}[9,22]$. Ni filling of the porous structure was achieved by pulsed electrodeposition at $310 \mathrm{~K}$ using a Watts bath with a carbon anode [9, 10, 12]. The electrodeposition was based on a repeated application of a negative pulse of $20 \mathrm{~V}$ during 8 $\mathrm{ms}$ followed by a positive pulse of $4 \mathrm{~V}$ for $2 \mathrm{~ms}$ to discharge the capacitor formed by the barrier layer. Each cycle ends with an $800 \mathrm{~ms}$ stage at $0 \mathrm{~V}$ for rearrangement of the electrolyte composition in the pores. A relatively large current is established due to displacement current effect and tunneling through the barrier layer during the abrupt rise of the voltage. As the voltage pulse is maintained, the displacement current decays to zero, but the tunneling is maintained.

After Ni deposition, the aluminum substrate was removed by chemical etching with a $\mathrm{HCl}-$ $\mathrm{CuCl}_{2}$ solution, and the composition, crystalline orientation and structure of the samples were characterized by $\mathrm{X}$-ray diffraction (XRD) and scanning electron microscopy (SEM).

The magnetic properties were studied using ferromagnetic resonance (FMR), vibrating sample magnetometry (VSM), and DC magnetometry with a SQUID. FMR experiments were carried out in a Bruker ESP 300 spectrometer operating at $v=9.39 \mathrm{GHz}$ with a cryogenic $T$ control using a $\mathrm{LN}_{2}$ reservoir that allowed varying $T$ from slightly above room temperature down to $\mathrm{T} \sim 120 \mathrm{~K}$. The recorded spectra contains a DPPH signal used for calibration that resonates near $\mathrm{H}=3355$ Oe. The experiments were performed varying $\mathrm{T}$ between $300 \mathrm{~K}$ and $120 \mathrm{~K}$, at two orientations of the magnetic field, parallel and perpendicular to the NW.

A SEM image of the AAO matrix with Ni NW deposited at the bottom of the pores, and a XRD diffraction scan are shown in Figure 1. 
The SEM image shows that the NW have a mean length of $(3.0 \pm 0.5) \mu m$ (the AAO membrane has a thickness of $20 \mu \mathrm{m}$ ). The XRD intensity ratio of the (220) and (111) Bragg reflections, when compared with that of Ni powder spectrum, gives evidence that there is no principal texture, but a soft preferential orientation along $<110>$. The broad peaks correspond to the amorphous alumina [20].

Figure 2 shows the longitudinal and transverse hysteresis loops measured at room $\mathrm{T}$ using VSM. The observed behavior, preserved in the whole T-range, indicates that the NW have uniaxial magnetic anisotropy with the easy axis parallel to the NW [23].

The effective anisotropy field in a uniaxial anisotropy model can be obtained from the magnetization curves $\mathrm{H} / / \mathrm{NW}$ and $\mathrm{H} \perp \mathrm{NW}$ (see Supplementary Material for details)

Figure 3 shows the FMR spectra at 120 and $300 \mathrm{~K}$. The anisotropy field $H_{A}$ can be determined from the resonance fields as

$$
H_{A}=-H_{\|}-\frac{H_{\perp}}{2}+\sqrt{\frac{5}{4} H_{\perp}^{2}+H_{\|} H_{\perp}}
$$

Details and additional data can be found in the Supplementary Material.

Figure 4 shows the results for the anisotropy field as a function of $T$ as measured from the FMR spectra, together with those obtained from the SQUID data (Supplementary Material).The two techniques give similar results indicating a significant reduction of the anisotropy field when decreasing $T$. 
A larger decrease of the anisotropy field with decreasing $T$ has been reported previously in $\mathrm{Ni} N W-A A O$ samples preserving the aluminum substrate $[13,19]$, and it has been attributed to magnetoelastic effects due to the mismatch between the thermal properties of aluminum and Ni NW-AAO, yet it was not directly measured.

In our case the massive aluminum substrate was removed, and our results have to be explained considering only the $\mathrm{Ni} \mathrm{NW}$ and their AAO matrix.

The anisotropy field would contain shape, magnetoelastic, and magnetocrystalline contributions [24]. We did not find evidence of the last contribution most likely due to the polycrystalline nature of the NWs. Due to the large aspect ratio of the NW, we can estimate the shape anisotropy field taking into account the contribution due to dipolar interactions between NW [25], as

$$
H_{\text {Ashape }}=2 \pi M_{s}(1-3 f)
$$

Here, $f=\frac{\pi}{2 \sqrt{3}}\left(\frac{d}{D}\right)^{2} \approx(0.10 \pm 0.01)$ is the filling factor, $d$ the pore diameter, $D$ the interpore distance, and $M_{S}$ the value of $\mathrm{Ni}$ saturation magnetization. At $300 \mathrm{~K}$, using $M_{s}=$ $488 \mathrm{emu} / \mathrm{cm}^{3}$ [24], and $f=0.10$ we calculate $H_{\text {Ashape }} \approx(2150 \pm 100)$ Oe using Eq (2). Our experimental data $H_{A}(300 \mathrm{~K})$ is in close agreement with this magnitude. This fact leads us to consider that at room $\mathrm{T}$ the shape anisotropy is the main contribution to the effective anisotropy field.

The saturation magnetization $M_{S}$ (measured in the same sample) increases $(6 \pm 1) \%$ when decreasing $\mathrm{T}$ from $300 \mathrm{~K}$ to $120 \mathrm{~K}$ (Supplementary Material). Since shape anisotropy is proportional to $M_{S}$, it is expected that $H_{\text {Ashape }}$ would have the same relative increase in this T-range, i.e.: $H_{\text {Ashape }}(120 \mathrm{~K})=(2270 \pm 100)$ Oe. In Figure 4 we included the shape anisotropy field, assuming it coincides with $H_{\text {Ashape }}$ at room $\mathrm{T}$. The experimental value of $H_{A}(120 K)$ is obtained by averaging the SQUID and FMR values. This average yields $H_{A}(120 \mathrm{~K})=(1860 \pm 60) O e$, smaller than $H_{\text {Ashape }}(120 \mathrm{~K})$, and points to a negative contribution to the anisotropy at $120 \mathrm{~K}: \Delta H_{A}=H_{A}(120 \mathrm{~K})-H_{\text {Ashape }}(120 \mathrm{~K})=-(410 \pm$ 120) Oe.

The angular dependent term of the uniaxial anisotropy energy density can be expressed [24] as $E(\theta)=K_{u} \sin ^{2} \theta$, where $\theta$ is the angle between the magnetization and the NW axis 
(easy axis). The constant $K_{u}$ is related to the magnetic anisotropy field through $K_{u}=\frac{H_{A} M_{\mathrm{s}}}{2}$. The previous calculations suggest that at low $\mathrm{T}$ there is an additional energy that can be estimated as:

$$
\Delta K_{u}=\frac{\Delta H_{A} M_{\mathrm{s}}(120 \mathrm{~K})}{2}=-(1.1 \pm 0.3) \times 10^{5} \frac{\mathrm{erg}}{\mathrm{cm}^{3}}
$$

This additional energy can be attributed to magnetoelastic effects due to the stress suffered by the Ni NW when T decreases.

In a previous study based on XRD measurements [20], we reported an average negative thermal expansion coefficient $\left\langle\alpha_{N i N W}\right\rangle=-(1.6 \pm 1.5) \times 10^{-6} \mathrm{~K}^{-1}$ along the NW axis for the Ni NW inside AAO, between $110 \mathrm{~K}$ and $350 \mathrm{~K}$. The analysis of this result [20] led us to conclude that the empty AAO matrix also has a negative thermal expansion in this Trange, opposite to the behavior of bulk alumina. Using this result, the experimental deformation of $\mathrm{Ni} \mathrm{NW}$ in $\mathrm{AAO}$ at $120 \mathrm{~K}$ relative to their equilibrium state at $310 \mathrm{~K}$ can be estimated as $\epsilon_{\|}=(3 \pm 3) \times 10^{-4}$.

What is the meaning of this result? Decreasing $\mathrm{T}$, isolated Ni NW would contract as bulk Ni does. But our result indicates an elongation, suggesting that Ni NW, adhered to the AAO pore walls, follow a behavior different from bulk $\mathrm{Ni}$. This fact can be explained considering that the expansion of the AAO matrix drags the NW through a shear stress, so they are also expanded along the NW long axis when the system cools. This assumption requires a strong enough bond between $\mathrm{Ni}$ and $\mathrm{AAO}$ pore walls, capable of resisting thermal cycling. Recalling the behavior of fiber composite materials [26], it can be shown that the shear on the NW walls causes a tensile stress $\sigma_{\|}$. Using the average Ni bulk thermal expansion coefficient between 120 and $300 \mathrm{~K},\left\langle\alpha_{\text {Nibulk }}\right\rangle=10.5 \times 10^{-6} K^{-1}[27]$, and the $\mathrm{Ni}$ polycrystalline Young modulus $E_{N i}=2.00 \times \frac{10^{12} \mathrm{dyn}}{\mathrm{cm}^{2}}$ [28], we can compute the stress in the NW axis direction at $120 \mathrm{~K}$ :

$$
\sigma_{\|}=E_{N i}\left[\epsilon_{\|}-\left\langle\alpha_{N i \text { bulk }}\right\rangle \Delta T\right]=(4.4 \pm 0.6) \times 10^{9} \frac{\mathrm{dyn}}{\mathrm{cm}^{2}}
$$

Following Coey [24] and Chikazumi [28] the uniaxial magnetoelastic anisotropy energy density $K_{u, m e}$ can be estimated as $K_{u, m e}=\frac{3}{2} \lambda\left(\sigma_{\|}\right)=-(2.2 \pm 0.3) \times 10^{5} \frac{\mathrm{erg}}{\mathrm{cm}^{3}}$ where $\lambda=$ $-34 \times 10^{-6}$ is the polycrystalline Ni magnetostrictive constant [28], if the axial stress is the only one acting on our system. This result is almost twice the value derived from the 
experiments in Eq. (3). Following Navas et al. and Dubois et al. $[13,18]$ we propose that a transversal stress is acting, leading to

$$
K_{u, m e}=\frac{3}{2} \lambda\left(\sigma_{\|}-\sigma_{\perp}\right)
$$

The transversal stress can be estimated by equating (3) and (5). In this way we obtain $\sigma_{\perp}=(2.3 \pm 0.9) \times 10^{9} \frac{d y n}{\mathrm{~cm}^{3}}$. This positive stress perpendicular to the NW axes has the same sign as $\sigma_{\|}$, which is consistent with an AAO matrix having an in-plane thermal expansion coefficient smaller than that of the Ni NW. Thus, the magnetic properties of the embedded nanowires, can be used as anisotropic stress sensor. The uncertainty $\Delta \sigma_{\perp}=0.9 \times 10^{9} \mathrm{dyn} /$

$\mathrm{cm}^{3}$ derives mainly from $\Delta \alpha_{N i N W}$. However, the sensitivity of this magnetoelastic sensor is linked to the ability to detect changes in the anisotropy field. Two consecutive FMR scans can be differentiated if the resonance field shifts by 10 Oe. This would imply resolving $\Delta \sigma \cong 5 \times 10^{7} \mathrm{dyn} / \mathrm{cm}^{2}(0.005 \mathrm{GPa})$ and strains of $\Delta \varepsilon \approx 3 \times 10^{-5}$ (Supplementary Material). Our results suggest that the thermal expansion of the AAO membrane has an anisotropic behavior.

In summary, we have presented structural and magnetic properties of self-supported nanocomposite membranes of nanoporous anodized alumina-Ni nanowires as a function of temperature. The Ni nanowires are ferromagnetic with easy magnetization axis parallel to the pore axis. We show by two independent techniques -FMR and magnetometry- that the measured anisotropy field of the nanowires decreases with decreasing $T$, opposite to the shape anisotropy (proportional to the magnetic moment of our samples). The departure of the anisotropy field from the shape anisotropy evolution with $T$ can be attributed to magnetoelastic effects, the majority of which can be accounted for by the tensile stress on the nanowires caused by the negative AAO expansion. The transversal stress is estimated to be positive at low $T$, smaller than the tensile stress along the nanowires. In this way, the Ni NW behave as nanometric stress sensors. Independent measurements of perpendicular strains in these systems would be necessary to corroborate this result.

\section{SUPPLEMENTARY MATERIAL}

MPMS SQUID measurements of magnetic moment as a function of temperature, magnetization curves used for determining the magnetic anisotropy, analysis of FMR 
spectra and their interpretation in terms of a uniaxial anisotropy field, AFM characterization, and estimation of sensitivity as differential stress sensor.

\section{ACKNOWLEDGMENTS}

This work was performed with grants of ANPCyT (PICT 2014 -1683, PICT 2015-0883), CONICET (PIP112-20110100895), Universidad Nacional del Litoral (CAI+D 50420150100108LI). L.F. holds a fellowship from CONICET; R.R.K. and A.M.G are researchers of CONICET.

\section{REFERENCES}

1. A. Fert, Rev. Mod. Phys. 80, 1517 (2008)

2. T. Vemulkar, R. Mansell, A. Fernández-Pacheco, and R. P. Cowburn, Adv. Funct. Mater. 26 4704 (2016).

3. M. Vázquez, Editor, Magnetic Nano- and Microwires: Design, Synthesis, Properties and Applications (Woodhead Publishing Series, 2015) ISBN 9780081001646.

4. C. Bran, E. Berganza, J. A. Fernández Roldán, E. M. Palmero, J. Meier, E. Calle, M. Jaafer, M. Foerster, L. Abahe, A. Farilmanse, R. P del Real, A. Asenjo, O. Chubykalo-Fesenko, M. Vázquez, ACS Nano 12, 5932 (2018).

5. Y.P. Ivanov, A. Chuvilin, S. Lopatin, and J. Kosel, ACS Nano 10, 5326 (2016).

6. F. Nasirpouri, S. Peighambari-Sattari, C. Bran, E. M. Palmero, E. Berganza Eguiarte, M. Vazquez, A. Patsopoulos and D. Kechrakos, Sci. Reports 9, 9010 (2019).

7. H. Lee, N.R. Hwang, S.H. Hwang, Y. Cho, Biosens. Bioelectron. 86, 864 (2016).

8. B. Özkale, N. Shamsudhin, G. Chatzipirpiridis, M. Hoop, F. Gramm, X. Chen, X, Martí, J.Sort, E. Pellicer, S. Pané, ACS Appl. Mater. Interfaces 7, 7389 (2015).

9. K. Nielsch, A. P. Müller, L. An-Ping, and U. Gösele, Adv. Mater. 12, 582 (2000).

10. D. Losic, A. Santos (Eds.) Nanoporous Alumina. Fabrication, Structure, Properties and Applications, Springer Series in Materials Science, Springer International Publishing, Switzerland (2015).

11. J. Guiliani, J. Cadena, and C. Monton, Nanotechnology 29, 075301 (2018).

12. A. Ramazani, M.A. Kashi, and G. Seyedi, J. Magn. Magn. Mater. 324, 1826 (2012). 
13. D. Navas, K. R. Pirota, P. Mendoza Zelis, D. Velázquez, C. A. Ross, and M. J. Vázquez, J. Appl. Phys. 103, 07D523 (2008).

14. R. López-Ruiz, C. Magén, F. Luis, and J. Bartolomé, J. Appl. Phys 112, 073906 (2012).

15. C. A. Ramos, E. Vassallo Brigneti, E. De Biasi, and M. Vázquez (2011). In: Nanowires Fundamental Research, Chapter 15. Abbass Hashim (Ed.), IntechOpen, (2011). ISBN: 978953-307-327-9.

16. D. Sellmyer, J.M. Zheng, and R. Skomski, J. Phys.: Condens. Matter 13, R433 (2001).

17. L. Piraux, G. Hamoir, A. Encinas, J. De La Torre Medina, F. A. Araujo, J. Appl. Phys. 114, 123907 (2013).

18. S. Dubois, J. Colin, J.L. Duvail, and L. Piraux, Phys. Rev. B 61, 14315 (2000).

19. C. T. Sousa, D. C. Leitao, M. P. Proenca, J. Ventura, A. M. Pereira, and J. P. Araujo, Appl. Phys. Rev. 1, 031102 (2014).

20. L. Forzani, C.A. Ramos, E. Vassallo Brigneti, A. M. Gennaro, and R. R. Koropecki, Appl. Phys. Lett.114, 111901 (2019).

21. H. Masuda and K. Fukuda, Science 268, 1466 (1995).

22. C. T. Sousa, D. C. Leitão, M. P. Proença, A. Apolinário, J. G. Correia, J. Ventura, and J. P. Araújo, Nanotechnology 22, 315602 (2011).

23. M. Vázquez, M. Hernández-Velez, K .Pirota, A. Asenjo, D. Navas, J. Velázquez, P. Vargas, and C. Ramos, Eur. Phys. J. B 40, 489 (2004).

24. J.M.D. Coey, Magnetism and Magnetic Materials, Cambridge University Press, New York, (2010).

25. A. Encinas-Oropesa, M. Demand, L. Piraux, I. Huynen, and U. Ebels, Phys Rev. B 63, 104415 (2001).

26. P. K. Mallick, Fiber reinforced composites, 3rd edition, chapter 3, CRC Press, New York (2007).

27. R. C. O'Handley, Modern Magnetic Materials: Principles and Applications, Wiley, New York (2000).

28. S. Chikazumi, Physics of Ferromagnets, $2^{\text {nd }}$ edition. Oxford University Press, (1997).

FIGURE CAPTIONS

Figure 1: Left: SEM lateral image. Right: XRD spectrum of a AAO-NW sample (black), and a simulated Ni powder spectrum (red). 
Figure 2: Magnetic moment as a function of the applied field $(\mathrm{H})$ at room temperature, with $\mathrm{H} / /$ and $\mathrm{H} \perp$ to the NW.

Figure 3: Ferromagnetic resonance of Ni NW arrays for $\mathrm{H} / / \mathrm{NW}$ and $\mathrm{H} \perp \mathrm{NW}$, obtained at $\mathrm{T}=120 \mathrm{~K}$ (blue) and $\mathrm{T}=300 \mathrm{~K}$ (red). The resonant fields $\mathrm{H}_{\|}$and $\mathrm{H}_{\perp}$ were obtained from the intercept of the absorption derivative with the high-field baseline.

Figure 4: Anisotropy field of Ni NW in freestanding porous alumina membranes as a function of temperature, determined from SQUID magnetometry (squares) and ferromagnetic resonance (circles). The solid line is $\mathrm{H}_{\text {Ashape }}$ as derived from the sample's magnetic moment $\mathrm{m}_{\mathrm{S}}(\mathrm{T})$, (Supplementary Material), and Eq (2) 


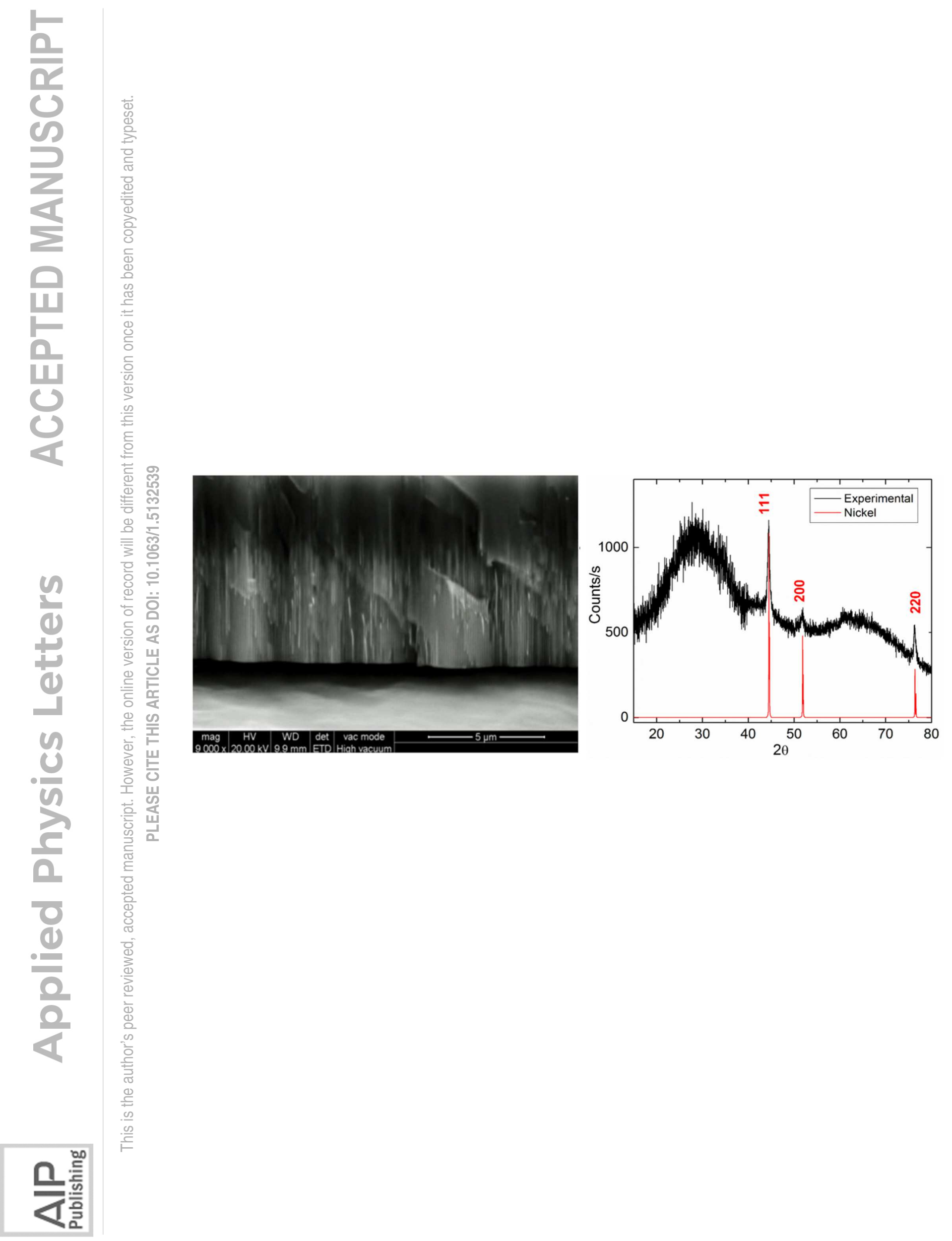




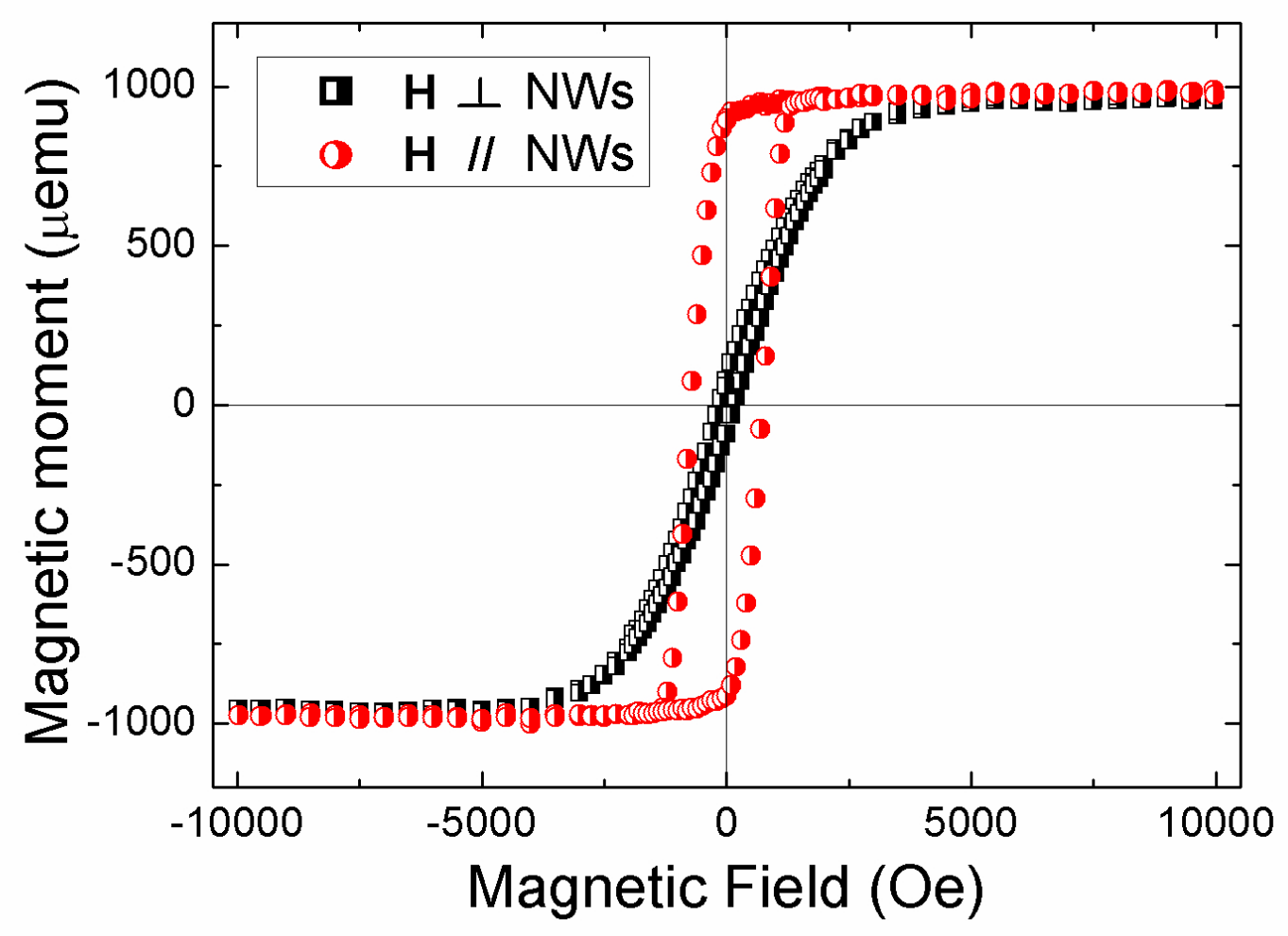




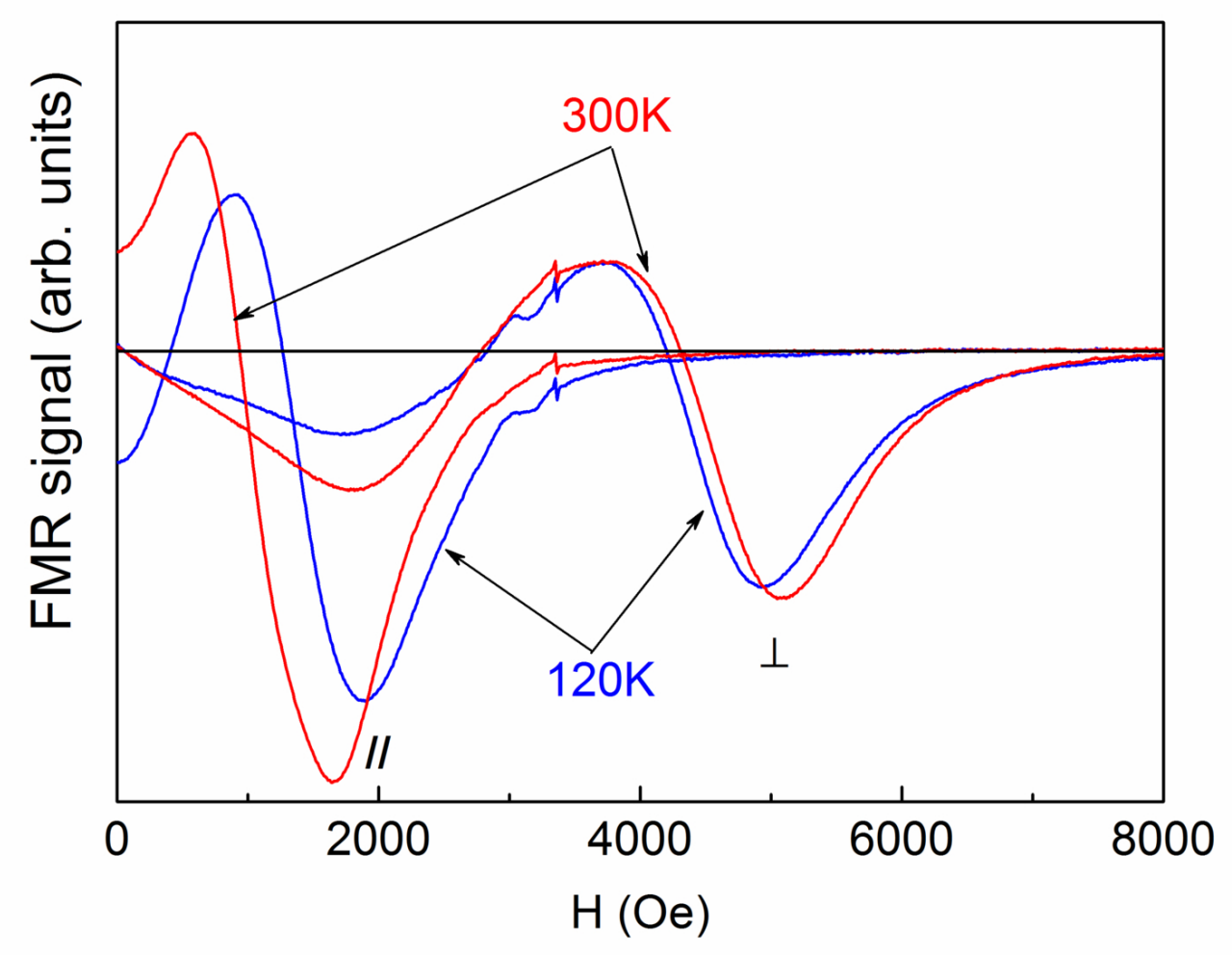

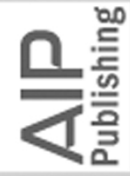




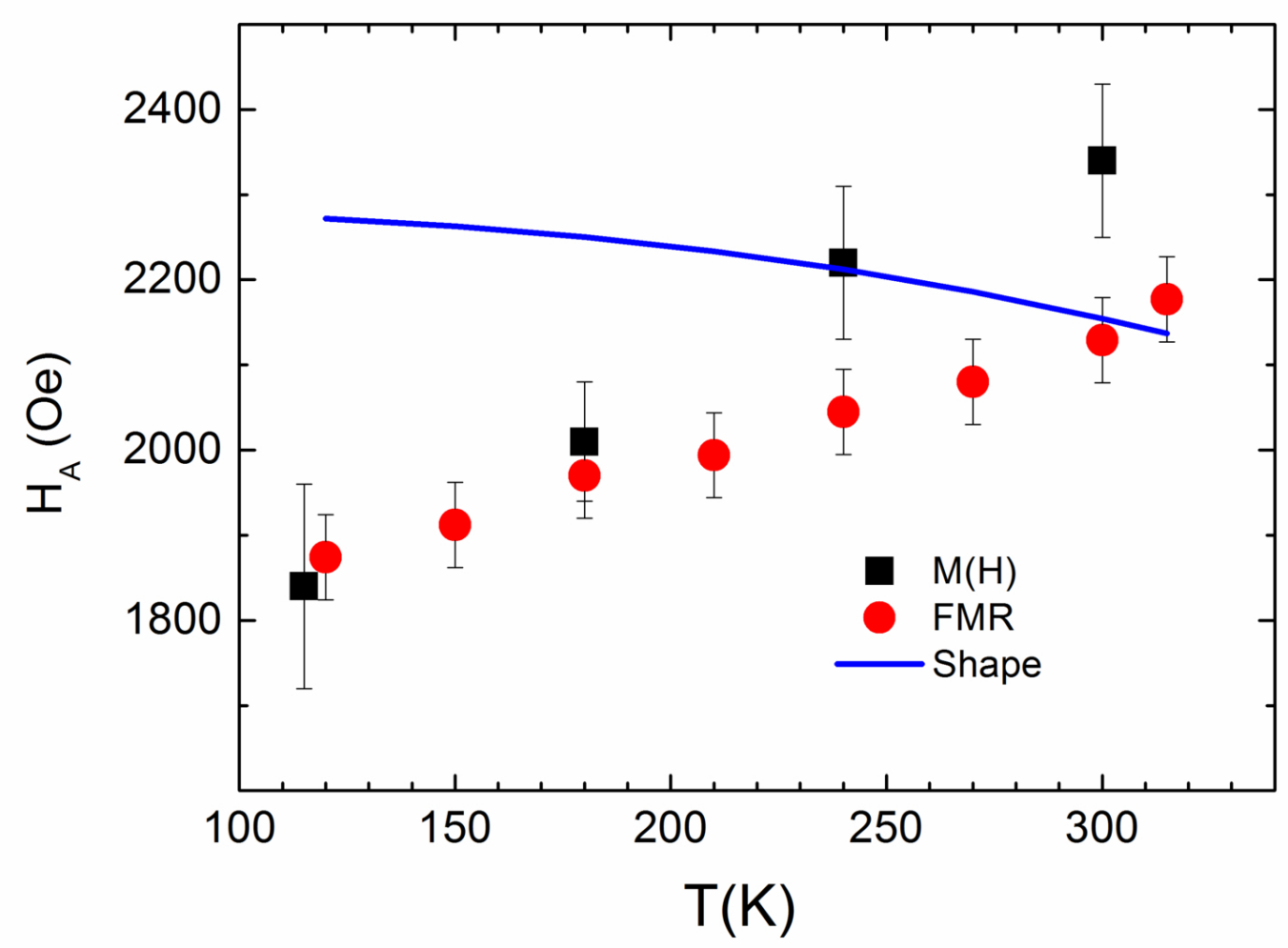

兄耪言 